Original Research Paper

\title{
On the General Solution of First-Kind Hypersingular Integral Equations
}

\author{
${ }^{1}$ Suzan J. Obaiys, ${ }^{1}$ Z. Abbas, ${ }^{2}$ N.M.A. Nik Long, ${ }^{2}$ A.F. Ahmad, ${ }^{2}$ A. Ahmedov and ${ }^{3}$ Haider Khaleel Raad \\ ${ }^{I}$ Department of Physics, Universiti Putra Malaysia, Serdang, Malaysia \\ ${ }^{2}$ Institute for Mathematical Research, Universiti Putra Malaysia, Serdang, Malaysia \\ ${ }^{3}$ Department of Physics, Xavier University, Cincinnati, Ohio, USA
}

Article history

Revised: $30-12-2015$

Accepted: 04-01-2016

Corresponding Author:

Suzan J. Obaiys

Department of Physics, Universiti

Putra Malaysia, Serdang, Malaysia

Email: suzan_ye@yahoo.com
Received: 10-11-2015

Abstract: A new algorithm is presented to provide a general solution for a first type Hyper singular Integral Equation (HSIE). The singular integral has been converted into a regular form by cancelling the singularity and then transforming it into a system of algebraic equation based orthogonal polynomials. We applied the convergence method presented by (Obaiys, 2013) to conform the numerical solution of the regularized Hadamard equation, which has shown an absolute agreement with the exact solution within small values of $n$. The proposed method has been examined by various HSIEs where the displacement function satisfies the Hölder-continuous first derivative. Such equations are particularly important inmany physics and engineering problems, such as fracture mechanics, acoustics and elasticity.

Keywords: HSIE, Singular Integrals, Fredholm Integral Equation, Chebyshev Polynomials

\section{Introduction}

\section{Basic Concepts}

One of the most frequently used numerical applications is the integral equation methods due to their ability to resolve the strong singularities that arise in stress fields where the boundary conditions change type (Lifanov et al., 2003; Iovane et al., 2003; Obaiys et al., 2012a). We present a new general solution for the first type HSIEs, whereas this approach depends on the analytical evaluation of the singular integral after the reduction for the higher order singularity. Integral equations arise in many physical problems, such as elasticity, acoustics and fracture mechanics which require the analytical solution of Fredholm integral equation (Martin and Rizzo, 1989; Martin, 1992; Obaiys et al., 2013):

$$
\int_{L} \lambda(t, x) g(t) d t=f(x), \quad x \in L
$$

where, $\lambda$ is a square integrable kernel of $(t, x)$ that has a logarithmic singularity on the diagonal $t=x$ :

$$
\iint_{L} \lambda^{2}(t, x) d t d x=B^{2}<\infty
$$

If $L$ is a piecewise smooth contour that includes the interval $[-1,1]$, (i.e.: Here we deal with an equation with Hilbert kernel). The regular part of the kernel $\lambda$ can be separated as singular and nonsingular parts by using the decomposing Fourier transform. Thus, by considering the segment $[-1,1]$ as a special case of $L$, Equation 1 becomes:

$$
\begin{aligned}
& \frac{1}{\pi} \int_{-1}^{1} K(t, x) g(t) d t \\
& +\int_{-1}^{1} G(t, x) g(t) d t=f(x), \quad x \in[-1,1]
\end{aligned}
$$

where, $G(t, x)$ is a regular kernel of $t$ and $x$ while the first kernel $K(t, x)$ has the form:

$$
K(t, x)=\frac{h(x)}{(t-x)^{\alpha}}, \quad \alpha \geq 2
$$

Which is called the hyper singular kernel and $K(x, x)$ $\neq 0$ and $h(t)$ is unknown function to be determined.

Furthermore $\alpha$ is the order of the integral which classifies its singularity. If $\alpha=2$, then Equation 3 becomes: 


$$
\begin{aligned}
& \frac{1}{\pi} \int_{-1}^{1} \frac{h(x) g(t)}{(t-x)^{2}} d t \\
& +\int_{-1}^{1} G(t, x) g(t) d t=f(x), \quad x \in[-1,1]
\end{aligned}
$$

A deep linked nexus of the different ordered singularities can be performed by the following exchangeability relationship (Martin and Rizzo, 1989):

$$
\int_{-1}^{1} \frac{g(t)}{(t-x)^{\alpha+1}} d t=\frac{1}{\pi} \int_{-1}^{1} \frac{\partial}{\partial x}\left(\frac{g(t)}{(t-x)^{\alpha}}\right) d t=\frac{1}{\alpha} \frac{d}{d x} \int_{-1}^{1} \frac{g(t)}{(t-x)^{\alpha}} d t
$$

For the second order singularity where $\alpha=1$, the above equation becomes:

$$
\int_{-1}^{1} \frac{g(t)}{(t-x)^{2}} d t=\frac{d}{d x} \int_{-1}^{1} \frac{g(t)}{t-x} d t, \quad x \in(-1,1)
$$

The right hand side of Equation 7 is called Cauchy Principle Value Integral (CPVI), which represents the base for ourapproximation and defined by (Iovane et al., 2003; Obaiys, 2013; Obaiys et al., 2013).

\section{Definition}

If a function $g(t), t \in[-1,1]$, be unbounded at some point $x \in(-1,1)$ and Riemann integrable over $[-1, x-\varepsilon) \subset[-$ $1, x)$ and $(x+\varepsilon, 1) \subset(x, 1], \forall \varepsilon>0$. Then CPVI of $g$ over [$1,1]$ is defined by:

$\frac{n=4}{x}$

Provided that this limit exists.

The CPVI in Equation 8 is a well-defined integral whenever $g$ is an improper Riemann integrable on $[-1,1]$, whilst a common CPVI is the Hilbert transform. The point $x$ is called a weak singularity of $g$ (Prem and Michael, 2005). It is important to know that once the CPVI has been approximated, the HSIEs can be obtained successfully by the consideration of the relationship in Equation 6 and it is defined as:

\section{Definition}

Lifanov et al. (2003) suppose that $g(x)$ is a real function defined on $[-1,1]$. Then:

$$
\int_{-1}^{1} \frac{g(t)}{(t-x)^{2}} d t=\lim _{\varepsilon \rightarrow 0}\left[\left(\int_{-1}^{x-\varepsilon}+\int_{x+\varepsilon}^{1}\right) \frac{g(t)}{(t-x)^{2}} d t-\frac{2 g(x)}{\varepsilon}\right]
$$

With existing and bounded limits of integration.
The choice of $g(t)$ in Equation 1 is significant and directly affects the singularity order where if $g(t)$ represents the slope function, then Equation 1 is a CPVI; whereas if it is chosen as a displacement function then a HSIE is performed.

\section{Preliminaries}

Numerous expansion of more advanced and efficient methods for the numerical solution of integral equations have been conducted (Mandal and Bhattacharya, 2007; Helsing, 2011). The HSIE of the form in Equation 5 where the regular kernel $G(t, x)=$ $0, h(x)=1$ and the displacement function $g$ satisfy the Hölder-continuous first derivative, becomes:

$\frac{1}{\pi} \int_{-1}^{1} \frac{g(t)}{(t-x)^{2}} d t=f(x), \quad-1 \leq x \leq 1$

Obviously $g$ represents the gap in the velocity potential of the flow across the plate. To ensure the continuity of the velocity at both endpoints $x= \pm 1$, the following boundary condition is important:

$g( \pm 1)=0$

Let the unknown function $g$ in Equation 10 be written as:

$$
g(t)=\sqrt{1-t^{2}} \varphi(t)
$$

where, $\varphi(x)$ is a well-defined function of $t$ and by substituting Equation 12 into Equation 10 with the use of the relationships in Equation 7, yield:

$$
f(x)=\frac{1}{\pi} \int_{-1}^{1} \sqrt{1-t^{2}} \frac{\varphi(t)}{(t-x)^{2}} d t=\frac{1}{\pi} \frac{d}{d x} \int_{-1}^{1} \sqrt{1-t^{2}} \frac{\varphi(t)}{t-x} d t
$$

To regularize the above CPVI, we add and subtract the value of $\varphi(x)$, which yields:

$$
f(x)=\frac{1}{\pi} \frac{d}{d x} \int_{-1}^{1} \sqrt{1-t^{2}}\left(\frac{\varphi(t)-\varphi(x)}{t-x}+\frac{\varphi(x)}{t-x}\right) d t
$$

Since:

$$
\int_{-1}^{1}\left(\sqrt{1-t^{2}} / \pi(x-t)\right) d t=x
$$

Results: 


$$
\begin{aligned}
& f(x)=\frac{1}{\pi} \frac{d}{d x}\left(\int_{-1}^{1} \sqrt{1-t^{2}} \frac{\varphi(t)-\varphi(x)}{t-x} d t-\pi x \varphi(x)\right) \\
& =\frac{1}{\pi} \int_{-1}^{1} \sqrt{1-t^{2}} \frac{-\varphi^{\prime}(t-x)+\varphi(t)-\varphi(x)}{(t-x)^{2}} d t-x \varphi^{\prime}(x)-\varphi(x) \\
& F(x)=\frac{1}{\pi} \int_{-1}^{1} \sqrt{1-t^{2}} \zeta(t, x) d t
\end{aligned}
$$

Where:

$$
\zeta(t, x)=\frac{-\varphi^{\prime}(t-x)+\varphi(t)-\varphi(x)}{(t-x)^{2}}
$$

And:

$$
F(x)=f(x)+x \varphi^{\prime}(x)+\varphi(x)
$$

The above integral in Equation 13 is a regularization of Equation 10 after subtracting its singularity where:

$$
\zeta(t, x) \equiv \varphi^{\prime \prime}(x) / 2 \text { when } t=x
$$

where, $\zeta$ is a real function belong to the class of Hölder on the set $[-1,1] \times[-1,1]$. It is known that HSIE in Equation 10 has the following exact solution (Mandal and Bhattacharya, 2007):

$$
g(x)= \begin{cases}\frac{1}{\pi} \int_{-1}^{1} f(t) \ln \left|\frac{x-t}{1-x t-\sqrt{\left(1-x^{2}\right)\left(1-t^{2}\right)}}\right| d t, \text { if } f(x) \neq 1, \\ \sqrt{1-x^{2}} & \text { if } f(x)=1 .\end{cases}
$$

\section{Approximation}

In computing integrals, there are several appropriate choices to select from. We developed a general approximating method for the bounded solution of any HSIE of the form in Equation 5, where $h(x)=1$ and $G(t, x) \neq 0$. Whereas the hyper singular kernel $K(t, x)$, the density function $g$ and the regular kernel $G(t, x)$ are supposed to be real functions belong to Hölder class on the sets $[-1,1] \times[-1,1],[-1,1]$ and $[-$ $1,1] \times[-1,1]$, respectively.

The function $\varphi(x)$ in Equation 12 approximated by a finite sum of an appropriate polynomial of degree $n$ :

$$
\varphi(x) \approx \sum_{i=0}^{n} C_{i} \zeta_{i}(x)
$$

Which means:

$$
g_{n}(x) \approx \sqrt{1-t^{2}} \sum_{i=0}^{n} C_{i} \zeta_{i}(x)
$$

And the condition:

$$
\int_{-1}^{1} g_{n}(t) d t=0
$$

Is desirable for the unique solution of $g$ while $C_{i}, i=$ $0,1,2, \ldots, n$, are the unknown coefficients to be determined, then Equation 5 becomes:

$\frac{1}{\pi} \sum_{i=0}^{n} C_{i}\left(\begin{array}{c}h(x) \int_{-1}^{1} \sqrt{1-t^{2}} \frac{\zeta_{i}(t)}{(t-x)^{2}} d t \\ +\pi \int_{-1}^{1} \sqrt{1-t^{2}} G(t, x) \zeta_{i}(t) d t\end{array}\right)=f(x)$

Both hyper singular and regular kernels in Equation 18 are approximated as follows:

$\frac{1}{(t-x)^{2}} \simeq \sum_{r=0}^{p} \rho_{r}(x) \zeta_{r}(t), \quad G(t, x) \simeq \sum_{q=0}^{s} k_{q}(x) \zeta_{q}(t)$

where, $\rho_{r}(x)$ and $k_{q}(x)$ are known expressions of $x$ and by substitute Equation 19 into Equation 18, yields:

$\frac{1}{\pi} \sum_{i=0}^{n} C_{i} Q_{i}\left(x_{j}\right)=f\left(x_{j}\right), \quad x \in[-1,1]$

Where:

$Q_{i}(x)=\int_{-1}^{1} \sqrt{1-t^{2}}\left(\begin{array}{c}h(x) \sum_{r=0}^{p} \rho_{r}(x) \zeta_{r+i}(t) \\ +\pi \sum_{q=0}^{s} k_{q}(x) \zeta_{q+i}(t)\end{array}\right) d t$

This approach reduces the integral equation problem in Equation 5 in to a finite linear algebraic system of $n+1$ linear equations with $n+1$ unknown coefficients $C_{i}$ of the form:

$\frac{1}{\pi} \sum_{i=0}^{n} C_{i} Q_{i}\left(x_{j}\right)=f\left(x_{j}\right), \quad j=0,1, \ldots, n$

where, $x_{j}$ to be chosen as the root of the polynomial $x_{j}$ on $[-1,1]$, i.e. $x=x_{j}$ and the coefficients $\left\{C_{i}\right\}_{i=0}^{n}$ satisfy (Obaiys, 2013):

$\left\langle K g_{n}+G g_{n}-f, g k(x)\right\rangle=0, \quad k=0,1,2, \ldots$ 
where, $K$ and $G$ are the singular and regular kernels defined in Equation 3 respectively and the calculation of $C_{i}$ endorses the evaluation of $g_{n}(t)$ in Equation 16.

For the error estimate of the HSIE of the first kind Equation 5, it is proven in (Obaiys, 2013) that if the functions $f \in C^{\ell}([-1,1])$ and $K(t, x) \in C^{\ell}([-1,1] \times[-1,1])$, then:

$$
\left\|g-g_{n}\right\|_{2, w}=C n^{-\ell}, \quad \ell \geq 1
$$

With $w=\sqrt{1-x^{2}}$. If $\ell$ can be chosen to be any large positive number, then the error in Equation 24 decreases very quickly and the convergence is very fast to the exact solution.

The numerical examples perform that for any singular point $\mathrm{x} \in[-1,1]$ the sequence $\left\{g_{n}\right\}$ converge uniformly in $L_{2, w}$ norm to $\{g\}$ as $n$ increases (Obaiys, 2013).

\section{Numerical Examples}

\section{Example 1}

If we consider $h(x)=1$ and $G(t, x)=0$ and $f(x)=1$, then Equation 5 takes the form:

$$
\frac{1}{\pi} \int_{-1}^{1} \frac{g(t)}{(t-x)^{2}} d t=1, \quad-1 \leq x \leq 1
$$

Which have the exact solution in Equation 14. The function $\zeta(x)$ is calculated numerically from the relationship in Equation 16 and $g(x)$ is approximately obtained. We can easily show the proof by considering $\zeta_{i}(x)$ in Equation 21 as Chebyshev polynomial of the second kind over the interval [-1,1]. It is known that orthogonal polynomials have a great variety and wealth of properties which playa great role and importance for quadrature methods as well as for the solution of mathematical and physical problems with a very efficient interpolation formula. The zeros of these polynomials are usually constant real, distinct and belong to a particular interval of $[-1,1]$ which conform with our research interest. These systems of zeros are used as nodes of the quadrature rules, which possess additional properties, like that of positivity orminimality of quadrature error. One can also obtain polynomials very close to the optimal one by expanding the given function in terms of Chebyshev polynomials and then cutting off the expansion at the desired degree. The second kind Chebyshev polynomial is defined as (Mason and Handscomb, 2003; Obaiys et al., 2012b):

$$
U_{i}(x)=\frac{\sin (i+1) \theta}{\sin \theta}, \cos \theta=x
$$

Where:

$U_{0}(x)=1, \quad U_{1}(x)=2 x, \quad U_{2}(x)=4 x^{2}-1$

Whereas the rest of the terms satisfy the following recurrence relationship:

$U_{i}(x)=2 x U_{i-1}(x)-U_{i-2}(x)$

And by taking the following cases for $\rho_{r}(x)$ and for $\rho_{q}(x)$ in Equation 35 defined below, as follows:

$$
\rho_{0}(x)=1, \quad \rho_{r}(x)=0 \quad \forall r>0, \quad \& G_{q}(x)=0 \forall q \geq 0
$$

Then $C_{0}=1$ and $C_{i}=0, \forall i>0$ and by substituting into Equation 12 for any value of $n$ gives:

$$
g(x)=\sqrt{1-x^{2}}
$$

Which performs Equation 14.

Table 1 shows that the approximate solutions coincide with the exact values.

\section{Example 2}

Consider the following HSIE:

$$
\begin{aligned}
& \frac{1}{\pi} \int_{-1}^{1} \frac{g(t)}{(t-x)^{2}} d t+\int_{-1}^{1} \cos (x) t^{4} g(t) d t \\
& =-5\left(16 x^{4}-12 x^{2}+1\right)-\frac{\cos (x)}{32}
\end{aligned}
$$

With the condition $g( \pm 1)=0$. The exact solution of Equation 29 is:

$$
g(x)=\left(16 x^{4}-12 x^{2}+1\right) \sqrt{1-x^{2}}
$$

The unknown function $g$ in Equation 29 is approximated by using the finite sum of Chebyshev polynomial of the second kind defined by (26), gives:

$$
g_{n}(t)=\sqrt{1-t^{2}} \sum_{i=0}^{n} C_{i} U_{i}(t)
$$

Table 1. The exact and approximate solutions of Equation 25

\begin{tabular}{lll}
\hline$\frac{n=4}{x}$ & Exact & Approximate \\
\hline \pm 1 & & \\
0 & 0.00 & 0.00 \\
\pm 0.2 & 1.00 & 1.00 \\
\pm 0.4 & 0.98 & 0.98 \\
\pm 0.6 & 0.92 & 0.92 \\
\pm 0.8 & 0.80 & 0.80 \\
\hline
\end{tabular}


Substitute Equation 31 into Equation 17 gives:

$\sum_{i=0}^{n} C_{i} \int_{-1}^{1} \sqrt{1-t^{2}} U_{i}(t) d t=0$

It is not difficult to show that (Mason and Handscomb, 2003):

$$
\int_{-1}^{1} \sqrt{1-t^{2}} U_{i}(t) d t=\int_{0}^{\pi} \sin (i+1) \theta \sin \theta d \theta= \begin{cases}\frac{\pi}{2} ; & i=0 \\ 0 ; & i \neq 0\end{cases}
$$

And by using Equation 33 into Equation 32, we obtain the value of the first coefficient:

$$
C_{0}=0
$$

The hyper singular and regular kernels in Equation 29 are approximated as follows (Obaiys et al., 2012b):

$$
\begin{aligned}
& \frac{1}{(t-x)^{2}}-2 \simeq \sum_{r=0}^{p}(r+1) U_{r}(x) U_{r}(t), \\
& G(t, x) \simeq \sum_{q=0}^{s} k_{q}(x) U_{q}(t)
\end{aligned}
$$

where, $U_{r}(x)$ and $k_{q}(x)$ are known expressions of $x$ and by substituting Equation 35 into Equation 29 and using the orthogonal property, yields:

$$
\begin{aligned}
& \sum_{i=0}^{n} C_{i}\left[-(i+1) U_{i}(x)+\frac{\pi}{2} k_{i}(x)\right] \\
& =-5\left(16 x^{4}-12 x^{2}+1\right)-\frac{\cos (x)}{32}
\end{aligned}
$$

And:

$$
k_{i}(x)=\frac{2}{\pi} \int_{-1}^{1} \sqrt{1-t^{2}} G(t, x) U_{i}(t) d t
$$

The linear system in Equation 37 can be evaluated analytically or numerically using a quadrature formula. By choosing the roots of $T_{n+1}(x)$ as the collocation points $x_{j}$ along the interval $[-1,1]$, which are:

$$
x_{j}=\cos \left(\frac{2 j-1}{2(n+1)} \pi\right), j=0,1, \ldots, n
$$

For $i=4$, we get:

$$
\begin{aligned}
& k_{0}(x)=\cos (x) / 8, k_{1}(x)=0, \quad k_{2}(x)=3 \cos (x) / 16 \\
& k_{3}(x)=0, k_{4}(x)=\cos (x) / 16, \quad k_{i}(x)=0 ; i \geq 5
\end{aligned}
$$

Substituting the values of Equation 37 into the system of Equation 36 for $n=4$, gives:

$$
\begin{aligned}
& \sum_{i=0}^{4} C_{i}\left[-(i+1) U_{i}\left(x_{j}\right)+\frac{\pi}{2} k_{i}\left(x_{j}\right)\right] \\
& =-5\left(16 x_{j}^{4}-12 x_{j}^{2}+1\right)-\frac{\cos \left(x_{j}\right)}{32}
\end{aligned}
$$

By solving the system Equation 40 for the unknown coefficients $C_{i} ; i=0,1, \ldots, 4$ and substituting the values of $C_{i}$ into Equation 31, we obtain the numerical solution of Equation 29, which is identical to the exact solution.

MATLAB codes are developed to obtain all the numerical results of Equation 29 where Table 2 presents the numerical experiment which perfectly agrees with the theoretical results.

\section{Example 3}

Consider the numerical solution of Fredholm integral equations of the first kind with a double pole singularity of the following form:

$\frac{1}{\pi} \int_{-1}^{1} \frac{g(t)}{(t-x)^{2}} d t+\frac{1}{\pi} \int_{-1}^{1} t x g(t) d t=$
$-64 x^{3}+17 x-8,-1 \leq x \leq 1$

The exact solution of Equation 41 is:

$$
g(x)=2 \sqrt{1-x^{2}}\left(x^{3}+\frac{1}{2}\right)
$$

We use the same steps explained in Example 2 where the roots are defined in Equation 38 and $i=4$ :

$$
\begin{aligned}
& \sum_{i=0}^{4} C_{i}\left[-(i+1) U_{i}(x)+\frac{\pi}{2} k_{i}(x)\right]= \\
& -64 x^{3}+17 x-8
\end{aligned}
$$

Table 2. The error of the numerical solution of Equation 29

\begin{tabular}{llll}
$\frac{n=4}{x}$ & Error & $\frac{n=16}{x}$ & Error \\
\hline-1 & $0.00 \mathrm{e}^{+000}$ & -1 & $0.00 \mathrm{e}^{+000}$ \\
-0.8 & $2.88 \mathrm{e}^{-007}$ & -0.8 & $2.88 \mathrm{e}^{-011}$ \\
-0.6 & $1.80 \mathrm{e}^{-007}$ & -0.6 & $1.26 \mathrm{e}^{-012}$ \\
-0.4 & $0.16 \mathrm{e}^{-008}$ & -0.4 & $2.13 \mathrm{e}^{-011}$ \\
-0.2 & $1.04 \mathrm{e}^{-007}$ & -0.2 & $2.41 \mathrm{e}^{-011}$ \\
0.0 & $2.82 \mathrm{e}^{-007}$ & 0.0 & $2.33 \mathrm{e}^{-012}$ \\
0.2 & $3.86 \mathrm{e}^{-007}$ & 0.2 & $3.19 \mathrm{e}^{-012}$ \\
0.4 & $2.85 \mathrm{e}^{-007}$ & 0.4 & $2.59 \mathrm{e}^{-011}$ \\
0.6 & $1.80 \mathrm{e}^{-007}$ & 0.6 & $0.94 \mathrm{e}^{-013}$ \\
0.8 & $2.88 \mathrm{e}^{-007}$ & 0.8 & $2.61 \mathrm{e}^{-011}$ \\
1 & $0.00 \mathrm{e}^{+000}$ & 1 & $0.00 \mathrm{e}^{+000}$ \\
\hline
\end{tabular}


Table 3. The error of the numerical solution of Equation 29

\begin{tabular}{llll}
\hline$\frac{n=4}{x}$ & Error & $\frac{n=32}{x}$ & Error \\
\hline-1 & $0.00 \mathrm{e}^{+000}$ & -1 & $0.00 \mathrm{e}^{+000}$ \\
-0.8 & $4.13 \mathrm{e}^{-005}$ & -0.8 & $3.27 \mathrm{e}^{-013}$ \\
-0.6 & $3.97 \mathrm{e}^{-005}$ & -0.6 & $2.36 \mathrm{e}^{-013}$ \\
-0.4 & $4.65 \mathrm{e}^{-005}$ & -0.4 & $1.54 \mathrm{e}^{-013}$ \\
-0.2 & $3.92 \mathrm{e}^{-005}$ & -0.2 & $2.45 \mathrm{e}^{-013}$ \\
0.0 & $2.89 \mathrm{e}^{-005}$ & 0.0 & $2.21 \mathrm{e}^{-013}$ \\
0.2 & $3.86 \mathrm{e}^{-005}$ & 0.2 & $3.19 \mathrm{e}^{-013}$ \\
0.4 & $2.85 \mathrm{e}^{-005}$ & 0.4 & $3.51 \mathrm{e}^{-013}$ \\
0.6 & $3.23 \mathrm{e}^{-005}$ & 0.6 & $0.93 \mathrm{e}^{-013}$ \\
0.8 & $4.28 \mathrm{e}^{-005}$ & 0.8 & $2.65 \mathrm{e}^{-013}$ \\
1 & $0.00 \mathrm{e}^{+000}$ & 1 & $0.00 \mathrm{e}^{+000}$ \\
\hline
\end{tabular}

The errors of the numerical solutions presented in Table 3 are computed as the absolute value of the difference between the exact and numerical solutions. Here, the simple solution of the linear system of equations provided a more efficient approximation and faster algorithm by increasing the values of $n$.

\section{Conclusion}

A general solution for any HSIE problem of the form in Equation 5 is presented. We reformulated the main integral problem as a set of linear algebraic equations that can be solved by applying the usual collocation method. Moreover, this numerical technique provided an efficient approximation algorithm that converge very fast to the exact solution even for small values of $n$. It can also be seen from the results that the error values based on Equation 24 decrease very fast for any singular point $x \in[-1,1]$ by increasing the values of $n$ and choosing an appropriate weight function. MATLAB12 codes are developed to obtain all the numerical results for different kernel functions and force functions $f(x)$ where the numerical experiments agree with the theoretical results.

\section{Acknowledgement}

The first author would like to thank the Universiti Putra Malaysia (UPM) for their support and providing facility accomplish this work.

\section{Author's Contributions}

Suzan J. Obaiys: Analyzing, deriving and writing of the manuscript.

Z. Abbas: Reviewing and analysing the manuscript.

N.M.A. Nik Long: Checking and analyzing the derive.

A.F. Ahmad: Design and data analysis.

A. Ahmedov: Analyze methematical derive.
Haider Khaleel Raad: Give the final review and approval for the manuscript to be submitted.

\section{Ethics}

This article is original and contains unpublished material. The corresponding author confirms that all of the other authors have read and approved the manuscript and no ethical issues involved.

\section{References}

Helsing, J., 2011. A fast stable solver for singular integral equations on piecewise smooth curves. SIAM J. Sci. Comput., 33: 153-174.

DOI: $10.1137 / 090779218$

Iovane, G., I.K. Lifanov and M.A. Sumbatyan, 2003. On direct numerical treatment of hypersingular integral equations arising in mechanics and acoustics. Actamechanica, 162: 99-110.

DOI: $10.1007 / \mathrm{s} 00707-002-1007-9$

Lifanov, I.K., L.N. Poltavskii and M.M. Vainikko, 2003. Hypersingular Integral Equations and their Applications. 1st Edn., CRC Press, London, ISBN-10: 0203402162, pp: 408.

Mandal, B.N. and S. Bhattacharya, 2007. Numerical solution of some classes of integral equations using Bernstein polynomials. Applied Math. Comput., 190: 1707-1716. DOI: $10.1016 /$ j.amc.2007.02.058

Martin, P.A., 1992. Exact Solution of a simple hypersingular integral equation. J. Integral Equat. Applic., 4: 197-204. DOI: $10.1216 /$ jiea/1181075681

Martin, P.A. and F.J. Rizzo, 1989. On boundary integral equations for crack problems. Proceedings of the Royal Society of London A: Mathematical, Physical and Engineering Sciences, (PES' 89), The Royal Society, pp: 341-355.

Mason, J.C. and D.C. Handscomb, 2003. Chebyshev Polynomials. 1st Edn., CRC Prress, ISBN-10: 1420036114, pp: 360.

Obaiys, S.J., 2013. On the convergence problem of onedimensional hypersingular integral equations. Math. Problems Eng., 2013: 974751-974756. DOI: $10.1155 / 2013 / 974751$

Obaiys, S.J., Z. Eshkuvatov and N.N. Long, 2013. On error estimation of automatic quadrature scheme for the evaluation of Hadamard integral of second order singularity. UPB Scientific Bull. Series A: Applied Math. Phys., 75: 85-98.

Obaiys, S.J., Z. Eskuvatov and N.N. Long, 2012a. A Chebyshev polynomial based quadrature for hypersingular integrals. AIP Conf. Proc., 1450: 139-141. DOI: 10.1063/1.4724130 
Obaiys, S.J., Z. Eskuvatov, N.N. Long and M.A. Jamaludin, 2012b. Galerkinmethod for the numerical solution of hypersingular integralequations based Chebyshev polynomials. Int. J. Math. Anal., 6: 2653-2664.

Prem, K. and R. Michael, 2005. Handbook of Computational Methods for Integration. 1st Edn., Chapman and Hall, CRC Press, Boca Raton,

ISBN-10: 1584884282, pp: 624. 